\title{
Viewing effects of 3-D images synthesized from a series of 2-D tomograms by VAP and HAP approaches
}

\author{
H. C. Zhai ${ }^{1}$, M. W. Wang ${ }^{1}$, F. M. Liu' ${ }^{1}$ Ken Y. Hsu ${ }^{2}$ \\ 1 Institute of Modern Optics, Nankai University, Tianjin 300071, China \\ Key Laboratory of Optoelectronic Information Science \& Technology, Ministry of Education, China \\ 2 Institute of Electro-Optical Engineering, National Chiao Tung University Hsinchu, Taiwan, China
}

\begin{abstract}
We report, for the first time, the experimental result and its analysis of synthesizing a series of simulating 2-D tomograms into a 3-D monochromatic image. Our result shows clearly the advantage in monochromaticity of a vertical area-partition (VAP) approach over a horizontal areapartition (HAP) approach during the final white-light reconstruction. This monochromaticity will ensure a 3-D image synthesis without any distortion in gray level or positional recovery.
\end{abstract}

Key words: Rainbow holography - 3-D monochromatic image - vertical area-partition - horizontal area-partition

\section{Introduction}

Two-step multiple-exposure rainbow holography can be employed to synthesize a 3-D image from a series of 2-D tomograms. In this technique, the horizontal area-partition (HAP) method was conventionally used to record the master hologram [1]-[5]. That is, the master hologram is horizontally area-partitioned into narrow slitted elementary master holograms, in which each 2-D tomogram obtained from the corresponding slice of the original object is recorded. During the white-light reconstruction, a 3-D image can be seen by the simultaneous read-out of the multiple holograms. However, different reconstructed images of the tomograms included in the 3-D image will be viewed in rainbow colors with some positional aberrations, if no special treatment is accompanied, which can easily result in false recovery in both perspective and gray level's judgment to human vision [6]. Some efforts have been made to alleviate these disadvantages [7] [8]. A dispersion compensation grating [9-10] has been employed to eliminate the color blur, and a method of inclining the master hologram [4]-[11] during the recording was proposed to improve the color blur effect. All these

Received 18 June 2002; accepted 15 September 2002.

Correspondence to: H. Zhai

Fax: ++86-22-23502974

E-mail: zhai@nankai.edu.cn methods need either complicated calculations or critical experimental conditions.

We formerly introduced a vertical area-partition (VAP) approach [12] for recording the master hologram. In this paper, we present, for the first time, the experimental result showing that, by employing this VAP approach, a monochromatic 3-D image composed of a series of reconstructed 2-D tomograms can be synthesized during the white-light reconstruction. Our experimental result also shows that, by the HAP approach, in contrast to VAP, the synthesized 3-D image is composed of a series of the reconstructed 2-D tomograms with rainbow colors, if no special treatment has been accompanied.

In section 2, two approaches, HAP and VAP, for recording the master hologram in the multiple-exposure rainbow holography are introduced, followed by the theoretical analysis and discussion of their different viewing effects in section 3. In section 4, experiments are presented and the results show the different viewing effects predicted by the theoretical analysis, before some conclusions are made in section 5 .

\section{HAP and VAP}

To understand the difference between HAP and VAP in recording the master holograms, we first briefly review the HAP and VAP approach in the two-step multiple-exposure rainbow holography for synthesizing a 3-D image from a series of 2-D tomograms.

Fig. 1 shows the optical layout of HAP and VAP approaches in the first step of the holographic recording. For HAP, the master hologram is divided into a series of vertical narrow elementary master holograms sitting side by side along the horizontal direction, with the neighboring elementary master holograms to record the successive frames of the 2-D tomograms (see fig. 1a). The master hologram $\mathrm{H}_{1}$ is located in the $x-y$ plane. A narrow vertical slit can be shifted in the $y$ direction, which sets a limit of region recording each elementary master hologram. An observation window 


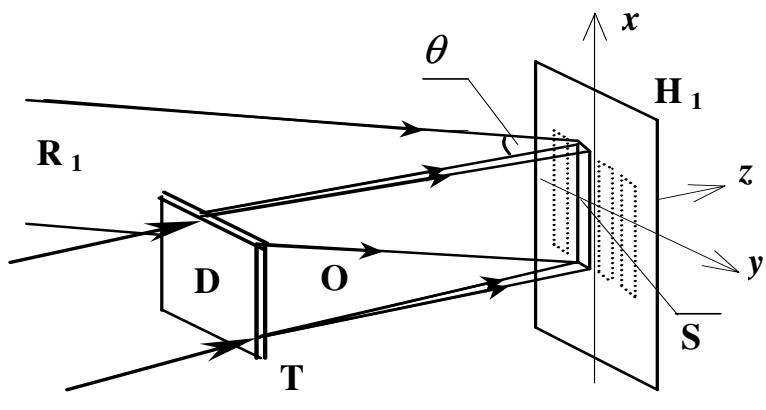

a)

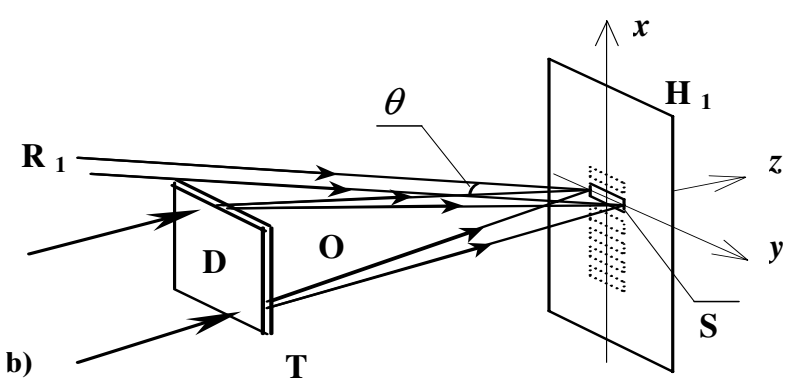

Fig. 1. Recording of slitted elementary master holograms: a) HAP; b) VAP. $H_{1}$ : master hologram, $S$ : slits, $\mathbf{R}_{1}$ : collimated recording reference beam, $\theta$ : angle between reference and object beam, D: diffuser, O: object beam, T: tomogram slice.

composed of all the real images of the slits during the white-light reconstruction determines the final viewing field.

In our VAP approach, instead, a horizontal slit is used to confine the recording region of each elementary master hologram (see fig. 1b). Different from that of the HAP approach, each slit has its length along the $y$ direction and can be shifted in the $x$ direction during the recording of the master hologram.

\section{Theoretical analysis of the viewing effects}

Because of the different constructions of the master holograms, the final viewing effects at the white-light reconstruction will be different, which can be understood as a result of the different relationships between the direction of dispersion and that of area-partition, as discussed in more detail in what follows.

In the optical systems for recording the master holograms, for either HAP or VAP, the distance between the input objects (i.e. the 2-D tomograms) and the master hologram $\mathrm{H}_{1}$ is much larger than the dimension of the slit. Hence, the primary wave vector of the object wave can be considered approximately to be along the $z$ direction. The plane wave reference beam, $R_{1}$, for recording all the tomograms is perpendicular to the $x$-axis. Consequently, the orientation of the interference fringes on the master hologram $\mathrm{H}_{1}$ is mainly along the $x$ direction, and the spacing of the fringes will depend on the angle $\theta$ between the normal of the reference wave vector and the $z$ axis. Since the conditions of the optical waves for recording $\mathrm{H}_{2}$, the second hologram of the two-step rainbow holography is similar to that for recording the master hologram $\mathrm{H}_{1}$, the fringe orientation on $\mathrm{H}_{2}$ is similar to that on $\mathrm{H}_{1}$.

Therefore, the dispersion of the reconstructed images during the final white-light reconstruction will spread mainly along $y$ and $z$ directions. The dispersion related to the wavelength, in the case of parallel reference beam, can be derived from the Champagne's imaging equation [12] as:

$$
\begin{aligned}
& x_{i}=x \\
& y_{i}=y-z \sin \theta^{\prime}\left[\left(\lambda_{0} / \lambda_{i}\right)-1\right] \\
& z_{i}=\left(\lambda_{0} / \lambda_{i}\right) z
\end{aligned}
$$

where $\theta^{\prime}$ is the angle of the final reconstruction reading beam, complementary to that of the final recording reference beam, $\lambda_{0}$ the optical wavelength used for the recordings, and $x, y$, and $z$ the corresponding coordinates of the reconstructed point object from $\mathrm{H}_{2}$ during the second recording, $\lambda_{i}$ represents any wavelength included in the white light during the final reconstruction, and $x_{i}, y_{i}$, and $z_{i}$ are the corresponding coordinates of the reconstructed image at the final white light reconstruction.

Because the slits for recording the elementary master holograms in HAP and VAP have different orientations, the read-out of the 3-D tomograms at the white light reconstruction will show different viewing effects.

By the HAP approach, an observation window composed of the reconstructed real images of the vertical slits at the final white-light reconstruction will disperse into a series of rainbow windows along the $y$ direction and $z$ direction as shown in fig. $2 \mathrm{a}$ and described in eqs. (1)-(3). Hence, when the reconstructed tomograms are viewed through a viewing line, $-z$ direction, for example, as shown in fig. $2 \mathrm{a}$, passing the dispersed real images of the correspondent slits with different wavelengths, rainbow colors distribution will be seen along the depth of the synthesized 3-D image. This may easily result in a false judgment of gray levels to human eyes, due to the human photopic eye response [5].

By the VAP approach, the dispersion effect of the reconstructed images will remain the same, that is, still mainly along $y$ and $z$ directions. It is, however, perpendicular to the direction of the area-partition, the $x$ direction. Therefore, along any possible viewing angle, all the reconstructed 2-D tomograms can be viewed simultaneously, through all the real images of the corresponding slits in one of the observation windows with the same wavelength, as shown in fig. $2 \mathrm{~b}$. Consequently, the rainbow effect will not appear among the reconstructed 2-D images at different depths of the synthesized 3-D image.

Besides, to view the reconstructed 3-D images of the tomograms through different windows along a viewing angle, false positional recovery may be viewed due to 


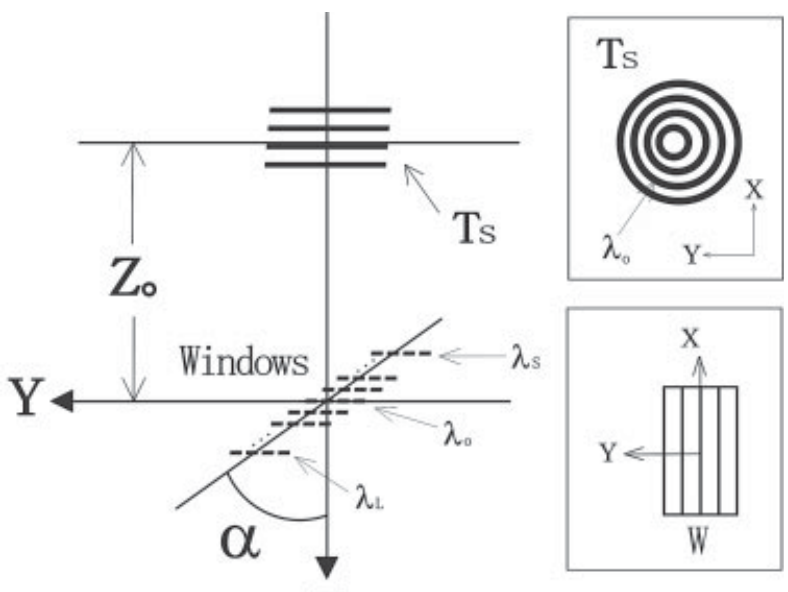

a)
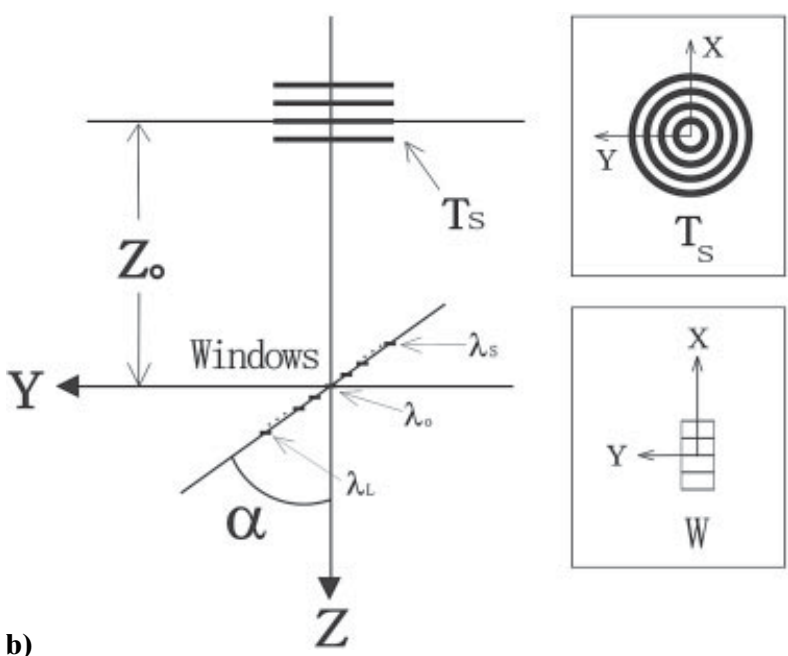

b)

Fig. 2. Observation windows in white-light reconstruction. a) HAP: Because of the rainbow distribution of the dispersed windows, rainbow colors and false recovery of position among the depths of the synthesized 3-D images will be viewed through different windows along $-z$ direction. b) VAP: When the reconstructed tomograms be viewed along $-z$ direction, the viewing line will pass only one single window with the identical wavelength, which ensure a monochromatic synthesis of the 3-D image. Ts: reconstructed tomograms (see fig. 3) as viewed along $-z$ direction, $Z_{0}$ : distance between the center of the reconstructed tomograms and the central window of $\lambda_{0}, \alpha$ : dispersion angle, W: construction of the observation window, $\lambda_{0}$ : wavelength same as in the recordings, $\lambda_{l}$ longer wavelength than in the recording, $\lambda_{s}$ : shorter wavelength than in the recording.

the relative distribution of the windows of the HAP approach (see fig. 2a), while in the VAP will not (see fig. 2b).

Eqs. (1)-(3) shows that the reconstructed image points have some shift in $y$ - and $z$-direction, but not in $x$-direction.. The shift is strongly $z$-coordinate and wavelength dependent. As to 3-D image synthesis from different 2-D slices, this kind of shift will result in a distortion of relative position of the whole 3-D image.
In HAP approach, the 3-D image white-light reconstructed will appear in different color $\left(\lambda_{i}\right)$ along the depth of the image, therefore, this kind of distortion will always exist. While in VAP approach, the wavelength will keep the same along the depth of the 3-D image. It is possible, therefore, to obtain a 3-D image through an appropriate viewing angle corresponding to the wavelength used in recording $\left(\lambda_{i}=\lambda_{0}\right)$. So that no relative positional distortion will be obtained in the VAP synthesis approach.

\section{Experimental result}

To compare the different viewing effects mentioned above at the final white-light reconstruction, specially designed 4 pieces of computer-simulated 2-D tomograms of circles (see fig. 3) with different diameters, with each circle simulating a 2-D tomogram at different depth, are used to synthesize a 3-D image. In the experiment, both the HAP and the VAP approaches were employed in the first recording to record the slitted elementary master holograms on $\mathrm{H}_{1}$, respectively.

In order to ensure a faithful comparison between the two approaches, identical experimental procedures were conducted for recording the second hologram $\mathrm{H}_{2}$ in the second step, where $\mathrm{H}_{1}$ was illuminated by a reference beam conjugate to that used for recording it. Thus, a series of real images of the 2-D tomograms were reconstructed from $\mathrm{H}_{1}$ and recorded on $\mathrm{H}_{2}$ by applying another recording reference beam onto it.

Coherent light of $532 \mathrm{~nm}$ from a Verdi laser was used for all the holographic recordings. All the holograms used were silver-halide emulsion plates of TJ-III type fabricated in Tianjin, China. In the recordings, the hologram plates were placed on the $x-y$ plane, and the angles between the reference beam and the $z$-axis were kept at $28^{\circ}$ or complementary to that. At the sec-
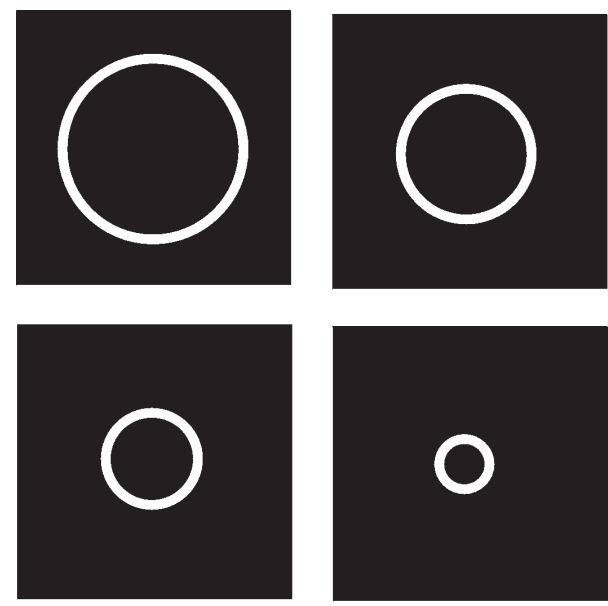

Fig. 3. 4 pieces of computer-simulated 2-D tomograms of circles, of which the diameters increase linearly along $z$-direction. 


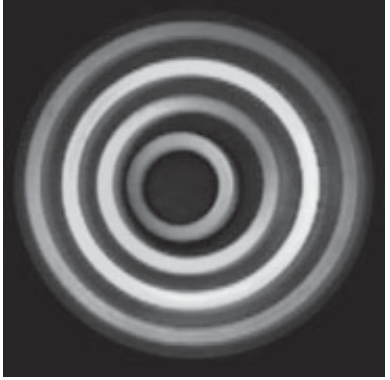

a)

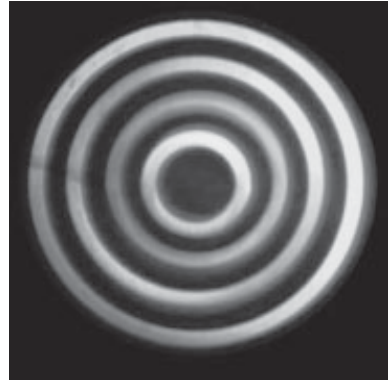

b)

Fig. 4. Reconstructed tomograms with rainbow color distribution by a) HAP approach and reconstructed monochromatic image of $\lambda_{0}$ by b) VAP approach.

ond recording, the second hologram $\mathrm{H}_{2}$ was recorded at a distance of $Z_{0}=500 \mathrm{~mm}$ away from the master hologram $\mathrm{H}_{1}$. This is the same distance between $\mathrm{H}_{1}$ and the center of the series of the original tomograms in the first recording. The final white-light reconstructed images by HAP approach is shown in fig. 4a, in which the reconstructed images at different depth of the 3-D image shows a rainbow-colored distribution and some positional distortion. Fig. $4 \mathrm{~b}$ shows the white-light reconstructed monochromatic images by VAP approach. As expected, no positional distortion appears there, for the wavelength is corresponding to that of recording. Reconstructed monochromatic images corresponding to other wavelengths $\lambda>\lambda_{0}$ and $\lambda<\lambda_{0}$ are also given in fig. $5 \mathrm{a}$ and fig. $5 \mathrm{~b}$, respectively, for comparison. Some inhomogeneity in intensity can be found in all the reconstructed monochromatic images, which is caused by else imperfect experimental condition, other than HAP and VAP themselves.

\section{Conclusions}

In conclusion, we have reported for the first time the experimental result and its analysis of the different viewing effects between VAP and HAP approaches in multiple-exposure rainbow holography for synthesizing a 3-D image from a series of 2-D tomograms. Our experimental results and analysis show clearly that any false recovery in gray levels or positions will not be produced within the viewing field of the synthesized 3-D image, without carrying out any extra experimental arrangement or complicated theoretical calculations.

The authors gratefully acknowledge the helpful discussion with Dr. P. Chavel and the supports from the Chinese National Foundation of Natural Science (No. 69677020) and the Tianjin Foundation of Natural

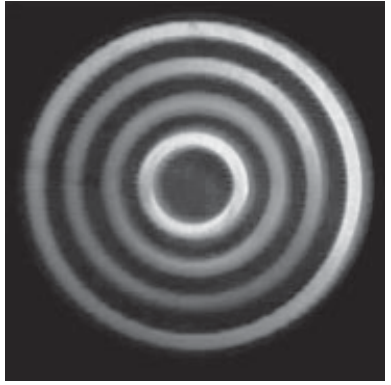

a)

Fig. 5. Reconstructed monochromatic tomograms by VAP approach of a) $\lambda>\lambda_{0}$ and b) $\lambda<\lambda_{0}$.

Science for this research. The research at NCTU is supported, in part, by a grant from the National Science Council (No. NSC89-2215-E-009-024).

\section{References}

[1] Johnson KM, Hesselink L, Goodman JW: Multiple exposure holographic display of CT medical data. Proc. SPIE 367 (1982) 149-154

[2] [Suzuki M, Kanaya M, Saito T: 3-dimentional illustration by multiply exposed hologram. Proc. SPIE 523 (1985) $38-41$

[3] Okada K, Ose T: Holographic three-dimensional display of X-ray tomogram. Proc. SPIE 673 (1986) 84-88

[4] Tsujiuchi J: Medical applications of holographic 3-D display. In Goodman JW (eds.): International trends in optics, pp 297-305. Academic Press Inc., New York 1991

[5] Xie JH, Zhao YL, Yu MW: Traversing area partition technique and its application in 2D/3D embossing holograms. Acta Optica Sinica 8 (1998) 410-416

[6] Jacobs GH: Comparative Color Vision. Academic Press Inc., New York 1981

[7] Guo SG, Zhai HC, Zhan YL et al : The distortion and its compensation of the reconstructed images in multiple-exposure rainbow hologram. Proc. ICO Topical Meeting of ICO'98, Optics for information infrastructure, Mu GG (ed.). JOEL 9 Supp. (1998) 214-217

[8] [Guo SG, Kang H, Zhan YL, Zhai HC, Bai B, Mu GG: Distortions and their compensation in 3-D holographic synthesis of multiple CT slides, J. Infrared Millim. Waves 19 (2000) 109-112

[9] Burckhardt CB: Display of holograms in white light. Bell Syst. Tech. J. 45 (1966) 1841-1850

[10] De Bitetto DJ: White light viewing of surface holograms by simple dispersion compensation. Appl. Phys. Lett. 9 (1966) 417-418

[11] Benton SA: Achromatic holographic stereograms (A). J. Opt. Soc. Am. 71 (1981) 1568

[12] Zhai HC, Wang MW et al: Vertical area partition of recording in the multiple-exposure rainbow hologram for 3-D achromatic synthesis of tomographic patterns Chinese J. Laser A 27 (2000) 828-832

[13] Champagne E: Nonparaxial image, magnification, and aberration properties in holography. J. Opt. Soc. Am. 57 (1967) 51-55 